\title{
Neutrinoless double $\beta$ decays of the top quark and other effects of heavy Majorana neutrinos
}

\section{Gad EILAM ${ }^{*}$}

Technion - Isreal Institute of Technology, Haifa, Israel

Email: eilamephysics.technion.ac.il

We discuss the rare decay of the top quark into a pair of same charge leptons (with identical or different flavors), a $b$ quark and a (real or virtual) $W^{-}$. The above process proceeds only if the exchanged neutrino $N$ is of the Majorana type. This decay is the neutrinoless double $\beta$ decay of the top. We find measurable values for its rate at the LHC with luminosity of $100 \mathrm{fb}^{-1}$. Furthermore, we consider an interaction of charged Higgs bosons with $N$ which leads to lepton number violating processes such as $p p \rightarrow \ell^{+} N \rightarrow \ell^{+} \ell^{+} H^{-}$, exhibiting spectacular events of the type $\ell^{+} \ell^{+} b \bar{b}+2$ jets.

Physics at LHC 2008

29 September - October 4, 2008

Split, Croatia

\footnotetext{
${ }^{*}$ Speaker.

${ }^{\dagger}$ I would like to thank my collaborators, especially Shaouly Bar-Shalom, for sharing their wisdom with me.
} 
The outline of this talk is as follows:

I will first motivate the study of heavy Majorana neutrinos and top quarks. Subsequently, Majorana neutrinos and lepton number violating (LNV) signals in $t$ quark and $W$ boson rare decays will be discussed. Then, I let $H^{+}$and $H^{-}$enter the game and briefly consider charged Higgs effects in the production and decay of a heavy Majorana neutrino at the Large Hadron Collider (LHC).

Let me present the motivation to study heavy Majorana neutrinos and top quarks. The discovery of neutrino oscillations implies that they have mass [1]. Thus indicating that there is New Physics (NP) beyond the Standard Model (SM). A simple way to consistently include sub-eV neutrinos into the SM, is to add superheavy right-handed neutrinos with GUT-scale masses and to rely on the seesaw mechanism [2] which yields a desired light neutrinos mass scale: $m_{V} \sim$ $M_{E W}^{2} / M_{G U T} \sim 10^{-2} \mathrm{eV}$. This seesaw mechanism links neutrino masses with NP at the GUT scale and raises the possibility that neutrinos will be of the Majorana type, where they are the antiparticles of themselves. The mechanism through which neutrinos acquire mass is yet unknown and might be different from the seesaw one. We take a purely phenomenological approach in which the masses of the heavy neutrinos are not predetermined by a specific model and will be taken here within the range explorable at the LHC [3].

Most of us believe that something new is lurking over there, at about the scale of EW symmetry breaking, which is around $250 \mathrm{GeV}$. The top quark at $172.6 \mathrm{GeV}$ [4] is the quark closest to that scale and is therefore the most sensitive to NP. For example, various models of NP may lead to Flavor Changing Neutral Currents (FCNC) of top, such as $t \rightarrow c H^{0}$ [5], which may be 10 orders of magnitude larger than their SM values [6].

The well known case which proceeds only if the light neutrino is Majorana, and is LNV with $\Delta L=2$, is the neutrinoless double beta decay $(0 v 2 \beta)$ [7] $(A, Z) \rightarrow(A, Z+2)+e^{-}+e^{-}$which has not been observed and it suffers from nuclear effects. Nevertheless, it produces very useful limits. Also interesting are the $\Delta L=2$, LNV processes in various high-energy collisions such as $e^{-} e^{-} \rightarrow W^{-} W^{-}$and in rare charged meson and lepton decays [8].

We explored [9] two additional LNV decay channels of the real top quark and of the real $\mathrm{W}$ boson, to like sign lepton pairs: $t \rightarrow b \ell_{i}^{+} \ell_{j}^{+} W^{-}$, and $W^{+} \rightarrow \ell_{i}^{+} \ell_{j}^{+} f \bar{f}^{\prime}$. The above top decay proceeds through the following diagrams:
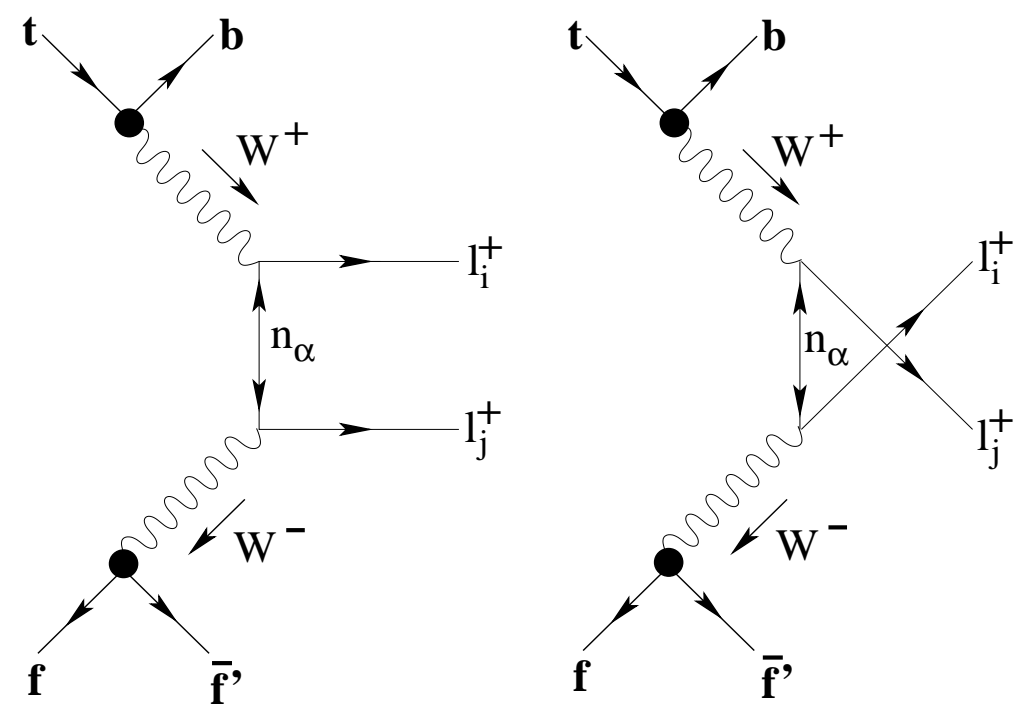
These decays are induced by heavy Majorana neutrino exchanges and may therefore serve as important tests of the neutrino sector and as a possible evidence for the existence of Majoranatype heavy neutrinos with masses at the EW scale. They both emanate from the same kernel: $W^{ \pm} W^{ \pm} \rightarrow \ell_{i}^{ \pm} \ell_{j}^{ \pm}$, with an exchange of a Majorana neutrino. This is the same kernel which induces $0 \vee 2 \beta$ in nuclei. However, in contrast to the nuclear case, the $0 v 2 \beta$ decays of $t$ and $W$ are dominated by the exchanges of heavy (EW scale) neutrinos instead of sub-eV neutrinos.

For the $t$ case the top-quark decays to an on-shell $W$ boson with a "wrong" charge, as compared with the "right" charge $W$ boson in the main decay $t \rightarrow b W^{+}$. The above "weird" $t$ and $W$ decays, originate from:

$$
\mathscr{L}=-\frac{g}{2 \sqrt{2}} B_{i n} W_{\mu}^{-} \ell_{i} \gamma^{\mu}\left(1-\gamma_{5}\right) n_{\alpha}+H . c .
$$

$\alpha=1-6$ stand for: 6 Majorana neutrino states, 3 of which are light and 3 are heavy. $B$ is a $3 \times 6$ matrix with elements $B_{i n} \equiv \sum_{k=1}^{3} V_{k i}^{L} U_{k n}^{*}$ where $V^{L}$ is the $3 \times 3$ unitary mixing matrix of the lefthanded charged leptons and $U$ is the $6 \times 6$ unitary mixing matrix in the neutrino sector.

The possibility of non-seesaw realizations or internal symmetries in the neutrino sector that decouple the heavy-to-light neutrino mixing from neutrino masses, cannot be excluded. In a model independent approach, the couplings are bounded by experimental constraints.

Assume: a single heavy neutrino $N$ dominates. The limits on its couplings to the charged leptons are expressed in terms of $\Omega_{\ell \ell^{\prime}} \equiv B_{\ell N} B_{\ell^{\prime} N}$.

Limits on its flavor-diagonal couplings from precision electroweak data, at 90\% CL, are [10]:

$$
\Omega_{e e} \leq 0.012, \Omega_{\mu \mu} \leq 0.0096, \Omega_{\tau \tau} \leq 0.016
$$

and on flavor-changing couplings, from limits on rare flavor-violating lepton decays:

$$
\left|\Omega_{e \mu}\right| \leq 0.0001,\left|\Omega_{e \tau}\right| \leq 0.02,\left|\Omega_{\mu \tau}\right| \leq 0.02
$$

The results of the calculations are depicted in the following figures:
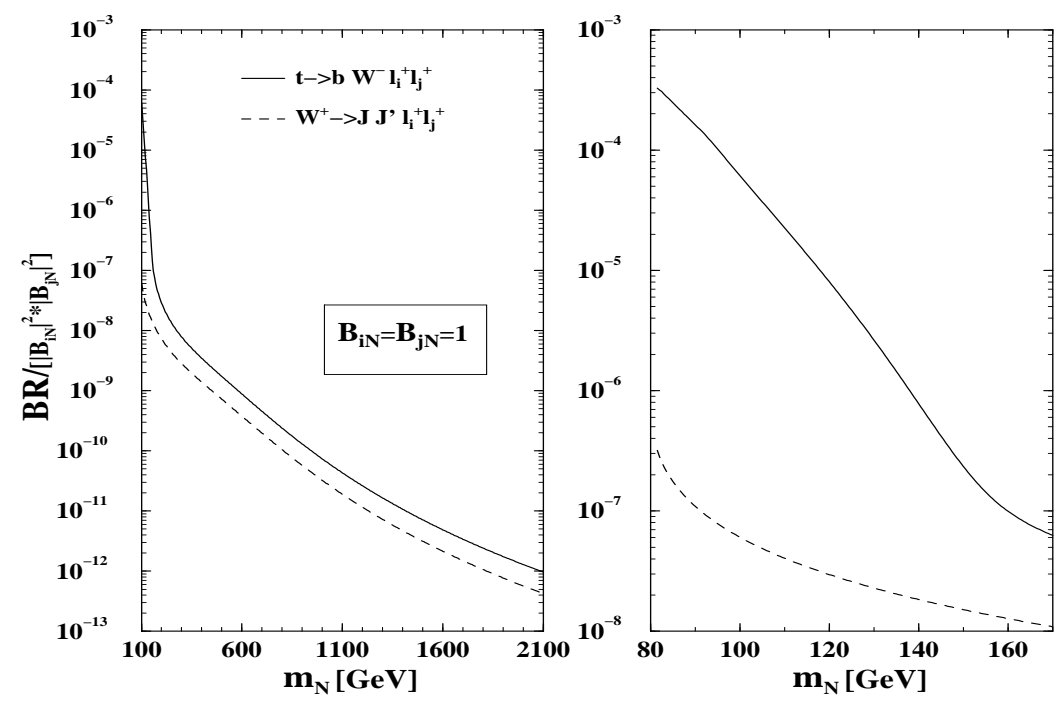
The branching ratios (BRs) here are scaled by the mixing parameters i.e., we took $B_{i N}=$ $B_{j N}=1$, and are shown as a function of $m_{N}$, the Majorana neutrino mass. We see that, for both decays, a sizable BR can arise only for $m_{N} \lesssim 100 \mathrm{GeV}$. Then: $B R\left(t \rightarrow b W^{-} \ell_{i}^{+} \ell^{+}\right) \sim 10^{-4}$ and $B R\left(W^{+} \rightarrow J \bar{J}^{\prime} \ell_{i}^{+} \ell_{j}^{+}\right) \sim 10^{-7}$.

For a more realistic BRs we use the bounds on the couplings. In case of the $W$-boson decay, the largest BR is of order of $10^{-10}$. This is too small to be observed at the LHC, where about $10^{9}-10^{10}$ inclusive on-shell $W^{\prime}$ 's are expected to be produced through $p p \rightarrow W+X$, at an integrated luminosity of $\mathscr{O}(100) \mathrm{fb}^{-1}$.

However, for more energetic off-shell $W$-bosons, produced at hadron colliders in the s-channel via $u d \rightarrow W^{*}$, the sensitivity to the heavy Majorana exchanges can be significantly enhanced [11]. Indeed, it was demonstrated that the s-channel $W^{*}$ can "decay" as $W^{*} \rightarrow 2$ jets $+\ell_{i}^{ \pm} \ell_{j}^{ \pm}$, by first decaying to an on-shell Majorana neutrino $W^{*} \rightarrow \ell N$, followed by $N \rightarrow \ell W \rightarrow \ell+2$ jets. This process is easily accessible at the LHC.

In the case of the top-quark decay $t \rightarrow b W^{-} \ell_{i}^{+} \ell_{j}^{+}$, taking $m_{N} \sim 100 \mathrm{GeV}$ and using the limits we discussed, the BRs for the various $\ell_{i}^{+} \ell_{j}^{+}$channels are given in the following table:

\begin{tabular}{c||c|c|c|c|c|c}
\hline \multicolumn{1}{c||}{} & \multicolumn{6}{c}{$B R\left(t \rightarrow b W^{-} \ell_{i}^{+} \ell_{j}^{+}\right) \times 10^{6}$} \\
$\ell_{i} \ell_{j}=$ & $e e$ & $\mu \mu$ & $\tau \tau$ & $e \mu$ & $e \tau$ & $\mu \tau$ \\
\hline$m_{N}=90 \mathrm{GeV}$ & 1.4 & 1.1 & 1.9 & $1.1 \cdot 10^{-4}$ & 1.6 & 1.4 \\
$m_{N}=100 \mathrm{GeV}$ & 0.6 & 0.5 & 0.8 & $0.4 \cdot 10^{-4}$ & 0.7 & 0.6 \\
\hline
\end{tabular}

The cross-section for $t \bar{t}$ production at $14 \mathrm{TeV}$ is $\sim 850 \mathrm{pb}$, yielding about $10^{8} t \bar{t}$ pairs at an integrated luminosity of $\mathscr{O}(100) \mathrm{fb}^{-1}$. Thus, a $B R\left(t \rightarrow b W^{-} \ell_{i}^{+} \ell_{j}^{+}\right) \sim 10^{-6}$ that can arise in most $\ell_{i}^{+} \ell_{j}^{+}$channels, should be accessible at the LHC. In particular, the flavor-changing decay channels $t \rightarrow b W^{-} e \tau$ and $t \rightarrow b W^{-} \mu \tau$ seem to be the most promising ones (in spite of the low $\tau$ detection efficiency) as the they are expected to be the cleanest with respect to background.

Note that a Majorana exchange is not necessarily the only mechanism leading to $\Delta L=2$ processes. One can envisage, for instance, a situation in which another type of new physics contributes together with the Majorana exchange. Viable examples are R-parity violating supersymmetry, or leptoquark exchange. In the (rather contrived) cases like these it is in principle possible to obtain destructive interference between the different mechanisms, thus evading the limits on the couplings considered here. Therefore, the rather sizable branching ratios, obtained for $\mathscr{O}(1)$ mixing angles cannot be excluded.

Now we change gear and let $\mathrm{H}^{+}$and $\mathrm{H}^{-}$enter the game, discussing charged Higgs-boson effects in the production and decay of a heavy Majorana neutrino at the LHC [12].

We considered a new interaction between a heavy Majorana neutrino and a charged Higgs boson,

$$
\mathscr{L}=\frac{g}{2 \sqrt{2}} \xi_{\ell N} \frac{m_{N}}{m_{W}} \bar{N}\left(1-\gamma_{5}\right) \ell H^{+}+H . c .
$$

where $\xi_{\ell N}$ are dimensionless parameters with sizes determined by the underlying NP.

We showed that $\mathscr{L}$ can have drastic implications on LNV signals with same-sign dileptons at the LHC. The LNV signal of heavy Majorana neutrinos previously considered at the LHC, $p p \rightarrow$ 
$\ell^{+} N \rightarrow \ell^{+} \ell^{+} W^{-}$, may be overwhelmed by $p p \rightarrow \ell^{+} N \rightarrow \ell^{+} \ell^{+} H^{-}$. With the subsequent decays $H^{-} \rightarrow \bar{t} b$ or $H^{-} \rightarrow W^{-} H^{0}$, the heavy Majorana neutrino production leads to the spectacular events $\ell^{+} \ell^{+} b \bar{b}+2$ jets.

We also explored the case $m_{N}<m_{H^{+}}$, where the decay $H^{+} \rightarrow \ell^{+} N$ can become the dominant $N$-production mechanism at the LHC. In particular, we show that the process $g \bar{b} \rightarrow \bar{t} H^{+}$followed by $t \rightarrow \bar{b} W^{-}$and $H^{+} \rightarrow \ell^{+} N \rightarrow \ell^{+} \ell^{+} W^{-}$could lead to another type of spectacular events of $\ell^{+} \ell^{+} b+$ 4 jets.

Now, a sentence to end with: Let us hope the LHC will be all in one i.e., that it will act both as a Higgs and as a top factory and that it will teach us about lepton number violation and ...

\section{References}

[1] For a recent review, see: B. Kayser, Neutrino mass, mixing, and flavor change, p. 163 in: Particle Data Group (A. Amsler et al.), Review of Particle Physics, Phys. Lett. B667 (2008) 1

[0804.1497[hep-ph]] and references therein.

[2] P. Minkowski, $\mu \rightarrow$ e $\gamma$ at a rate of one out of 1-billion muon decays? Phys. Lett. B67 (1977) 421; T. Yanagida, Horizontal symmetry and masses of neutrinos, in proceedings of Workshop on the unified theory and the baryon number in the universe, edited by A. Sawada and A. Sugamoto, KEK, Tsukuba, Japan, 1979, p. 95; M. Gell-Mann, P. Ramond and R. Slansky, Complex spinors and unified theories, in proceedings of Supergravity, edited by P. van Nieuwenhuizen and D.Z. Freedman, North Holland, 1979, p. 315; R.N. Mohapatra G. and Senjanovic, Neutrino mass and spontaneous parity nonconservation, Phys. Rev. Lett. 44 (1980) 912.

[3] For an example of such a model see: A. Pilaftsis, Radiatively induced neutrino masses and large Higgs neutrino couplings in the standard model with Majorana fields, Z. Phys. C 55 (1992) 275.

[4] For this most recent result see: F. Garberson (CDF and D0 collaborations), Top quark mass and cross section results from the Tevatron, 0808.0273 [hep-ex].

[5] For a recent review see: J. M. Yang, Probing new physics from top quark processes at LHC: A mini review, Int. J. Mod. Phys. A 23 (2008) 3343 [0801 . 0210 [ hep-ph] ] and references therein.

[6] G. Eilam, J. L. Hewett and A. Soni, Rare decays of the top quark in the standard and two Higgs doublet models, Phys. Rev. D 44 (1991) 1473 [Erratum-ibid. D 59 (1999) 039901]; B. Mele, S. Petrarca and A. Soddu, A new evaluation of the $t \rightarrow c H$ decay width in the standard model, Phys. Lett. B 435 (1998) 401 [hep-ph / 98054 98].

[7] For a recent review see: P. Vogel, Neutrino mass and neutrinoless double beta decay, in proceedings of 4th international workshop on neutrino oscillations In Venice: Ten years after the neutrino oscillations edited by M. Baldo Ceolin, Papergraf, Padova, 2008, p. 75 [0807.1559 [hep-ph]] and references therein.

[8] For an extensive list of references for these and other processes involving neutrinos at the EW scale, see: A. Atre, T. Han, S. Pascoli and B. Zhang, The Search for Heavy Majorana Neutrinos, 0901.3589 [hep-ph].

[9] S. Bar-Shalom, N. G. Deshpande, G. Eilam, J. Jiang and A. Soni, Majorana neutrinos and lepton-number-violating signals in top-quark and W-boson rare decays Phys. Lett. B 643 (2006) 342 [hep-ph / 0608309 ]; Recently, a calculation similar to ours appeared, leading to similar conclusions: Z. Si and K. Wang, GeV Majorana neutrinos in top-quark decay at the LHC, Phys. Rev. D 79 (2009) 014034 [0810. 5266 [hep-ph] ]. 
[10] S. Bergmann and A. Kagan, Z-induced FCNC and their effects on neutrino oscillations, Nucl. Phys. B $\mathbf{5 3 8}$ (1999) 368 [hep-ph/9803305].

[11] F. M. L. Almeida, Y. D. A. Coutinho, J. A. Martins Simoes and M. A. B. do Vale, Signature for heavy Majorana neutrinos in hadronic collisions, Phys. Rev. D 62 (2000) 075004 [hep-ph/ 000202 4]; T. Han and B. Zhang, Signatures for Majorana neutrinos at hadron colliders, Phys. Rev. Lett. 97 (2006) 171804 [hep-ph/0 604064 ].

[12] S. Bar-Shalom, G. Eilam, T. Han and A. Soni, Charged Higgs boson effects in the production and decay of a heavy Majorana neutrino at the LHC, Phys. Rev. D 77 (2008) 115019 [0803.2835 [hep-ph]]. 